\title{
ON THE NUMBER OF LATIN RECTANGLES
}

\author{
DOUGLAS S. STONES
}

(Received 24 March 2010)

2000 Mathematics subject classification: primary 05B15; secondary 05A05, 11B50, 20D60, $20 \mathrm{D} 45$.

Keywords and phrases: Latin square, Latin rectangle, autotopism, automorphism, Alon-Tarsi conjecture, quasigroup, orthomorphism.

\section{The number of Latin rectangles}

A Latin rectangle $L$ is a $k \times n$ array with symbols from $\mathbb{Z}_{n}$ such that each row and each column contains only distinct symbols. When $k=n, L$ is called a Latin square. We say $L$ is reduced if the first row is $(0,1, \ldots, n-1)$ and the first column is $(0,1, \ldots, k-1)^{T}$. The number of $k \times n$ Latin rectangles, denoted $L_{k, n}$, is related to the number of reduced $k \times n$ Latin rectangles, denoted $R_{k, n}$, by the formula $L_{k, n}=n !(n-1) ! R_{k, n} /(n-k)$ !. We also write $R_{n}=R_{n, n}$. McKay and Wanless [10] gave $R_{k, n}$ when $n \leq 11$.

The author's thesis [14] primarily investigates the number $R_{k, n}$. For example, we use a formula of Doyle [6,12] to find $R_{4, n}$ for $n \leq 80, R_{5, n}$ for $n \leq 25$ and

- $\quad R_{6,12}=16790769154925929673725062021120$ and

- $\quad R_{6,13}=4453330421956050777867897829494620160$.

In general, the problem of finding $R_{k, n}$ is difficult and, furthermore, the literature contains many published errors (see $[5,9,10,12]$ for surveys of its history). In addition to tackling the enumeration problem computationally, we also find theoretical results for $R_{k, n}$. For example, we find the value of $R_{k, n} \bmod n$ for all $k$ and $n$ [19].

THEOREM 1.1. If $k \geq 1$ and $n \geq 1$, then $R_{k, n} \equiv\left((-1)^{k-1}(k-1) !\right)^{n-1} \bmod n$.

Thesis submitted to Monash University, November 2009. Degree approved, February 2009. Main supervisor: Dr Ian M. Wanless. Secondary supervisor: Associate Professor Graham Farr. External supervisor: Professor Darryn Bryant.

Supported by the Faculty of Science Dean's Postgraduate Research Scholarship and Postgraduate Publications Award.

(c) 2010 Australian Mathematical Publishing Association Inc. 0004-9727/2010 \$16.00 
Theorem 1.1 implies the surprising fact that $R_{n} \bmod n$ is an indicator variable for primality of $n$. We also generalize recurrence congruences for $R_{3, n}$ by Riordan [11] and Carlitz [3] to arbitrary fixed $k$. The techniques were further developed to encompass the number of certain graph factorizations and the size of certain subsets of Latin hypercuboids (a very broad generalization of Latin rectangles).

\section{Orthomorphisms and partial orthomorphisms}

A partial orthomorphism of $\mathbb{Z}_{n}$ is an injection $v: S \rightarrow \mathbb{Z}_{n}$ for some $S \subseteq \mathbb{Z}_{n}$ such that $i \mapsto v(i)-i$ is also an injection [20]. An orthomorphism is a partial orthomorphism with $|S|=n$ [8]. Let $z_{n}$ be the number of orthomorphisms $\sigma$ of $\mathbb{Z}_{n}$ for which $\sigma(0)=0$. We extend a result by Clark and Lewis [4] who found $z_{n} \bmod n$ for prime $n$ [16].

THEOREM 2.1. $R_{n+1} \equiv z_{n} \equiv-2 \bmod n$ for odd prime $n$ and $R_{n+1} \equiv z_{n} \equiv 0 \bmod n$ for composite $n$.

The enumeration of partial orthomorphisms is also linked to the value of $R_{k, n}$ [17]. We give new sufficient conditions for when a partial orthomorphism admits a completion to an orthomorphism and give a method for finding the number of partial orthomorphisms with $|S|=a$, for fixed $a$ [17].

Let $d$ be a divisor of $n$. If $\sigma$ is an orthomorphism of $\mathbb{Z}_{n}$ such that $\sigma(i) \equiv \sigma(j) \bmod d$ whenever $i \equiv j \bmod d$ then we call $\sigma$ a $d$-compound orthomorphism. We develop the theory of $d$-compound orthomorphisms and, in particular, two special subclasses, compatible and polynomial orthomorphisms [16].

\section{The Alon-Tarsi conjecture}

The sign of a Latin square is -1 if it has an odd number of rows and columns that are odd permutations, otherwise it is +1 . Let $R_{n}^{\text {EVEN }}$ and $R_{n}^{\text {ODD }}$ be respectively the number of Latin squares of order $n$ with sign +1 and -1 . The Alon-Tarsi conjecture asserts that $R_{n}^{\text {EVEN }} \neq R_{n}^{\text {ODD }}$ when $n$ is even [1]. In a 1997 paper, Drisko [7] proved that $R_{n+1}^{\mathrm{EVEN}} \not \equiv R_{n+1}^{\mathrm{ODD}} \bmod n$ for prime $n$ and suggested some ideas for future research in the study of the Alon-Tarsi conjecture, which we show to be futile with the following theorem [18].

THEOREM 3.1. If $2 \leq t \leq n$, then $R_{n+1}^{\mathrm{EVEN}} \not \equiv R_{n+1}^{\mathrm{ODD}} \bmod t$ if and only if $t=n$ is prime.

\section{Autotopisms and subsquares}

We also investigate symmetries of Latin squares; see [5] for the relevant definitions. Autotopisms and automorphisms play a key role in finding divisors of $R_{n}$. Moreover, Latin squares that admit automorphisms typically contain partial orthomorphisms. Let $L$ be a Latin square of order $n$ and let $\operatorname{Atop}(L)$ be the autotopism group of $L$. We bound the maximum cardinality of $\operatorname{Atop}(L)$, enabling us to find divisors of $R_{n}$ for large $n$. A similar method gives a bound on the maximum number of $k \times k$ subsquares in a Latin square, for general $k$. 
THEOREM 4.1. If $L$ is a Latin square of order $n$, then

$$
|\operatorname{Atop}(L)| \leq n^{2} \prod_{t=1}^{\left\lfloor\log _{2} n\right\rfloor}\left(n-2^{t-1}\right) .
$$

THEOREM 4.2. The number of $k \times k$ subsquares in a Latin square of order $n$ is $O\left(n^{\left\lceil\log _{2}(\lfloor k / 2\rfloor+1)\right\rceil+2}\right)$.

Finally, we find new strong necessary conditions for when an isotopism is an autotopism of some Latin square [15]. We use $\Xi_{n}$ to denote the set of permutations $\alpha \in S_{n}$, such that $(\alpha, \alpha, \alpha)$ is an automorphism of some Latin square of order $n$.

THEOREM 4.3. Suppose that $\alpha \in S_{n}$ has precisely $m$ nontrivial cycles of length $d$. If $\alpha$ has no fixed points, then $\alpha \in \Xi_{n}$ if and only if $m$ is even or $d$ is odd. If $\alpha$ has at least one fixed point, then $\alpha \in \Xi_{n}$ if and only if $n \leq 2 m d$.

THEOREM 4.4. Suppose $\alpha \in S_{n}$ consists of a $d_{1}$-cycle, a $d_{2}$-cycle and $d_{\infty}$ fixed points. If $d_{1}=d_{2}$ then $\alpha \in \Xi_{n}$ if and only if $0 \leq d_{\infty} \leq 2 d_{1}$. If $d_{1}>d_{2}$ then $\alpha \in \Xi_{n}$ if and only if:
(a) $d_{2}$ divides $d_{1}$;
(b) $d_{1} \geq\lceil n / 2\rceil$;
(c) $d_{2} \geq d_{\infty}$; and
(d) if $d_{2}$ is even then $d_{\infty}>0$.

Theorems 4.3 and 4.4 generalize a theorem of Wanless [20] (see also [2]) for cyclic automorphisms. The techniques developed in [15] were subsequently used to study the parity of the number of quasigroups [13].

\section{References}

[1] N. Alon and M. Tarsi, 'Colorings and orientations of graphs', Combinatorica 12(2) (1992), 125-134.

[2] D. Bryant, M. Buchanan and I. M. Wanless, 'The spectrum for quasigroups with cyclic automorphisms and additional symmetries', Discrete Math. 304(4) (2009), 821-833.

[3] L. Carlitz, 'Congruences connected with three-line Latin rectangles', Proc. Amer. Math. Soc. 4(1) (1953), 9-11.

[4] D. Clark and J. T. Lewis, 'Transversals of cyclic Latin squares', Congr. Numer. 128 (1997), $113-120$.

[5] J. Denés and A. D. Keedwell, Latin Squares and their Applications (Academic Press, New York, 1974).

[6] P. G. Doyle, 'The number of Latin rectangles', arXiv:math/0703896v1 [math.CO], 15 pp.

[7] A. A. Drisko, 'On the number of even and odd Latin squares of order $p+1$ ', Adv. Math. 128(1) (1997), 20-35.

[8] A. B. Evans, Orthomorphism Graphs of Groups (Springer, Berlin, 1992).

[9] B. D. McKay, A. Meynert and W. Myrvold, 'Small Latin squares, quasigroups and loops', J. Combin. Des. 15 (2007), 98-119.

[10] B. D. McKay and I. M. Wanless, 'On the number of Latin squares', Ann. Comb. 9 (2005), 335-344.

[11] J. Riordan, 'A recurrence relation for three-line Latin rectangles', Amer. Math. Monthly 59(3) (1952), 159-162. 
[12] D. S. Stones, 'The many formulae for the number of Latin rectangles', Electron. J. Combin. 17 (2010), A1.

[13] D. S. Stones, 'The parity of the number of quasigroups', submitted.

[14] D. S. Stones, 'On the number of Latin rectangles'. PhD Thesis, Monash University, 2010.

[15] D. S. Stones, P. Vojtěchovský and I. M. Wanless, 'Autotopisms and automorphisms of Latin squares', in preparation (working title).

[16] D. S. Stones and I. M. Wanless, 'Compound orthomorphisms of the cyclic group', Finite Fields Appl. 16 (2010), 277-289.

[17] D. S. Stones and I. M. Wanless, 'A congruence connecting Latin rectangles and partial orthomorphisms', submitted.

[18] D. S. Stones and I. M. Wanless, 'How not to prove the Alon-Tarsi conjecture', submitted.

[19] D. S. Stones and I. M. Wanless, 'Divisors of the number of Latin rectangles', J. Combin. Theory Ser. A 117(2) (2010), 204-215.

[20] I. M. Wanless, 'Diagonally cyclic Latin squares', European J. Combin. 25 (2004), 393-413.

DOUGLAS S. STONES, School of Mathematical Sciences, Monash University, Vic 3800, Australia

e-mail: the_empty_element@yahoo.com 